\title{
Growth Visualization of Chinese Fir based on 3-PG Model
}

\author{
L.Y. Ma, H.Q. ZHANG \\ Research Institute of Forestry Resource Information Techniques, Chinese Academy of Forestry \\ Beijing, China
}

\begin{abstract}
Stand visualization is an important means of realistic simulation of stand structure, prediction for future stand growth status and manual operation. For long-term dynamic visual expression of stand, it is required to take advantage of physiological and ecological model and build the whole stand growth model. There is growing worldwide interest in the use of 3-PG model. This study took fir plantation as the research object, and used the 3-PG model to predict future stand characteristics. The simulation system was based on C\# language, .NET frame and DIRECTX 9.0, achieving a two-dimensional and three-dimensional visual simulation of stand growth. The results showed: 3-PG model can effectively predict fir stand growth effectively, and visual simulation technology can make stand growth more intuitive and efficient expression in order to provide a visual decision platform to improve forest management.
\end{abstract}

\section{Keywords-stand visualization; 3-PG model; stand growth}

\section{INTRODUCTION}

The visual simulation of stand is based on the stand growth model and computer graphics-based approach. Forest information can be more intuitive, more detailed manifested through the computer. It can present true performance of real structural characteristics and growth conditions, providing an intuitive and accurate criterion for the forest management decision-making [1]. The perfect growth models have a great significance to predict changes of the forest and build stand structure and growth visualization system. Thus, the use of a reasonable and perfect model can accurately predict the true trend of forest dynamic. The realistic forest dynamic simulation plays a more intuitive and accurate guidance on forest management [2]. Traditional stand growth visualization set a fixed growth rate. The major strength of this empirical approach is in describing the best relationship between the measured data and the growth-determining variables using a specified mathematical function or curve. It may be an appropriate method for predicting short-term yield for time scales over which historical growth conditions are not expected to change significantly.Now more and more growth models are to reflect the relationship between the environment, structure and forest growth. Researchers are to extend the model to more closely represent biophysical processes and adapting the model so that it is more relevant to management questions.

There is a growing worldwide interest in the use of 3-PG model. Previously, the 3PG model has been successfully parameterized and tested across the globe in predicting forest productivity in Eucalyptus globulus and E. grandis. The 3PG model requires few input parameters and readily available soils and climate data to model the annual (and monthly) growth and stand development of forest stands [3]. We can parameterize the 3PG model for a specific tree species or a hybrid. Therefore, this use will entail its adaptation to an increasing range of species. Now, predicting stand biomass with 3PG has been done for Chinese Fir due to the handling of the problems with the assignment of species-specific parameters. M.C. Guo applied 3PG to estimate the stand growth of Chinese fir plantations at the experimental plots of subtropical forest experimental center, and proved the model was with high accuracy and reliability to act as an useful tool for the management of Chinese fir plantation.[4] 3PG growth predictions for Chinese Fir could be of great advantage to researchers, landowners and forest managers, and help make effective management decision support tool of forest dynamics simulation as well as management for fir stand resources. However, the 3PG model is seldom applied in the forest visualization system. To resolve this issue and provide a good visualization for the growth and yield prediction of the stands in varying growing conditions, the objective of this paper is to present a new approach for handling the stand growth simulation with the applying of 3PG model to the simulation system, leading to a more realistic simulation of the stand growth dynamics.

\section{STUDY AREA}

This study area is located in Huangfengqiao, the state-owned forest farms in You County, Hunan Province, about 115-1270 $\mathrm{m}$ above sea level, characterized by the humid subtropical monsoon climate with abundant light and heat resources (lat. 27.04 '-27.06'N, long. $130^{\circ} 04$ 'to $113^{\circ} 43^{\prime} \mathrm{E}$ )[5]. This study was carried out in fixed sample in pure Fir artificial stands, collecting the 2008 survey data and Hunan Youxian Station 2008 meteorological data; Model validation data were taken from the each wooden survey data for this plot in 2013.

\section{RESEARCH METHODS}

\section{A. 3-PG models}

3-PG is a simple, process-based, stand-level model of forest growth developed by Landsberg and Waring. It requires only readily available site, climatic data and genetic characteristics of trees as inputs and predicts the time-course of stand development on a monthly basis in a form familiar to the forest manager. It uses a series of models to simulate the carbon balance, water balance, and solar radiation changes, 
and then simulate the dynamic growth stands. Due to the small amount of input parameters of the model and easy to get, it has been applied to different species of forestry research in many countries and regions. Almeida used 3-PG model as a management decision support tool of a large area of fast-growing Eucalyptus stands in Brazil. The 3-PG model parameters were calibrated to predict the existing production as well as new estimated production capacity[6]. Coops developed simulation software 3-PGS to support remote sensing data, using remote sensing satellite images as input information, which you can get a large area stands climate, forest leaf area index data, which greatly enhanced the 3-PG to predict large-scale forest dynamic[7]. 3-PG, effective management decision support tool of forest dynamics simulation development as well as management of forestry resources, is with high flexibility, adaptability, accuracy and a high value.

\section{B. Visual Simulation Technology}

Stand visualization system is based on Visual Studio 2008 platform, using the C\# language and Direct 3D technology, and it applies 3-PG model to explain the forest growth.

1) Direct 3D: Direct 3D (D3D) is based on Microsoft's Common Object Mode(COM), 3D graphics API. It may bypass the graphical display interface (GDI) and directly support various underlying hardware operation of the API, greatly improving the speed of graphics processing [8]. D3D Device is divided into the hardware abstraction layer device (HAL Device) and reference test equipment device (REF Device), providing these main functions of matrix transformation, three-dimensional image rendering and realization display. Upon completion of the coordinate transformation, lighting, and texture mapping, Direct3D then draws configuration values in the color buffer, and finally the color buffer contents can be displayed on the screen through a graphical refresh. The realization of three-dimensional display in this system is by means of Direct3D graphics manipulation techniques.

2) The visual simulation system: The system uses four-tier structure, namely the system layer, development layer, application layer and the presentation layer. System layer, including the development of the necessary hardware and operating system conditions; development layer offer environment and tools to support system development, responsible for the development of fir shape model and program development; The application layer is for user actions and system operations, providing the results to the presentation layer through a series of calculations according to the various types of models and parameter settings; presentation layer is the user interface, providing an interface for human-computer interaction, responsible for displaying data and receiving data entered by the user.

\section{RESULTS AND ANALYSIS}

\section{A. Data Input}

The growth simulation parameters input include: meteorological data, stand data and time data. Meteorological data was on a monthly step length, and it mainly included monthly solar radiation, rainfall, water vapor pressure, maximum temperature, minimum temperature, whether extreme weather and the number of days. Table 1 is Hunan Youxian weather station data on a monthly step in 2009,detailed recording the required model variables.Figure1,2respectivelyshow the stand data input interface and species modified parameters input interface.

TABLE I. WEATHER OBSERVATION DATA FROM YOU COUNTYHUNAN PROVINCE IN 2009

\begin{tabular}{ccccccc}
\hline Month & RAD & Rainfall & VPD & Tmax & Tmin & FrostDays \\
\hline 1 & 756.7 & 64.7 & 6 & 6.4 & 1.0 & 3 \\
2 & 831.3 & 80.9 & 6.5 & 9.8 & 2.8 & 2 \\
3 & 1002.4 & 191.6 & 12.1 & 20.8 & 11.4 & 1 \\
4 & 1400.4 & 139.7 & 16.1 & 23.6 & 16.2 & 0 \\
5 & 1811.4 & 154 & 20.3 & 29.5 & 20.9 & 0 \\
6 & 1944.2 & 177.8 & 24.7 & 30.7 & 23.4 & 0 \\
7 & 2873.6 & 115.8 & 26.3 & 35.1 & 26.6 & 0 \\
8 & 2701.4 & 99.7 & 27 & 34.3 & 25.8 & 0 \\
9 & 1834.5 & 49.2 & 23.9 & 31.2 & 22.4 & 0 \\
10 & 1529.9 & 58.1 & 17.5 & 26.4 & 17.7 & 1 \\
11 & 1167.8 & 164.1 & 11.3 & 18.6 & 10.5 & 2 \\
12 & 1013.2 & 25.3 & 7.4 & 15.9 & 0.42 & 3 \\
\hline
\end{tabular}

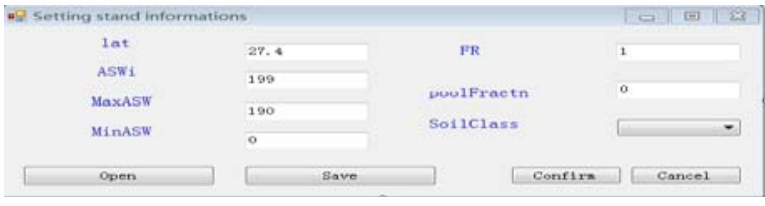

FIGURE I．STAND DATA INPUT INTERFACE

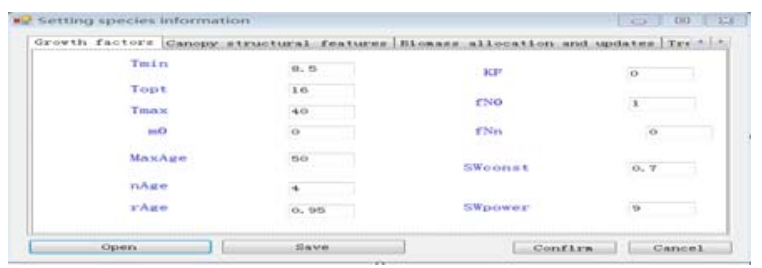

FIGURE II. THE SPECIES MODIFIED PARAMETERS INPUT INTERFACE

\section{B. The Simulation Results}

With 3PG model and the existing model parameters, the forest growth forecast can be achieved. Using the attribute information of per tree (location, diameter, height, crown width), calculating tree competition, then each tree growth and the amount allocated to per tree were obtained according to their competition. And stand growth visual can be achieved. Finally, compare, analyze every tree growth forecast and actual growth, and evaluate predictive modeling results. The output results were per tree diameter, height growth. Figure 3 is the regression analysis results of 2013 per plant simulated $\mathrm{DBH}$, height and measured values. As can be seen, $\mathrm{DBH}$, tree height simulated and observed values showed a highly significant linear correlation $(\mathrm{P}<0.001)$. Analog values can explain more than $80 \%$ of the corresponding change in the 
observed values, indicating that the model is valid for the expression of fir stands physiological growth. Table 2 shows the simulation results of 3-PG model and corresponding observed values in 2013. The results show that the average accuracies of the model are more than $90 \%$.

TABLE II.SIMULATION RESULTS OF THE DBH AND HEIGHT FOR CHINESE FIR IN 2013

\begin{tabular}{|c|c|c|c|c|c|c|}
\hline \multirow[t]{2}{*}{ Num } & \multicolumn{2}{|l|}{$\mathrm{DBH}$} & \multirow{2}{*}{$\begin{array}{c}\text { Accuracy } \\
\qquad \%\end{array}$} & \multicolumn{2}{|l|}{$\mathrm{H}$} & \multirow[b]{2}{*}{$\begin{array}{c}\text { - Accuracy } \\
\qquad \%\end{array}$} \\
\hline & $\begin{array}{l}\text { measured } \\
/ \mathrm{cm}\end{array}$ & $\begin{array}{c}\text { estimated } \\
/ \mathrm{cm}\end{array}$ & & $\begin{array}{c}\text { measured } \\
/ \mathrm{m}\end{array}$ & $\begin{array}{l}\text { estimated } \\
/ \mathrm{m}\end{array}$ & \\
\hline 1 & 19.25 & 19.03 & 98.2 & 11.4 & 11.0 & 96.5 \\
\hline 2 & 18.20 & 17.84 & 97 & 12.2 & 10.6 & 87.0 \\
\hline 3 & 10.20 & 10.76 & 94.2 & 6.7 & 7.2 & 92.5 \\
\hline 4 & 15.10 & 16.20 & 92.7 & 10.9 & 10.1 & 93.4 \\
\hline 5 & 19.00 & 18.14 & 95.2 & 12.0 & 10.9 & 90.8 \\
\hline 6 & 15.70 & 16.78 & 92.3 & 10.9 & 10.3 & 94.5 \\
\hline 7 & 16.00 & 16.91 & 93.7 & 10.8 & 10.1 & 93.5 \\
\hline 8 & 19.70 & 18.81 & 94.5 & 11.2 & 10.0 & 89.3 \\
\hline 9 & 18.90 & 17.83 & 93.5 & 11.0 & 10.6 & 96.5 \\
\hline 10 & 20.70 & 19.09 & 91.1 & 12.0 & 10.1 & 84.2 \\
\hline$\ldots$ & $\ldots$ & $\ldots$ & $\ldots$ & $\ldots$ & $\ldots$ & $\ldots$ \\
\hline
\end{tabular}

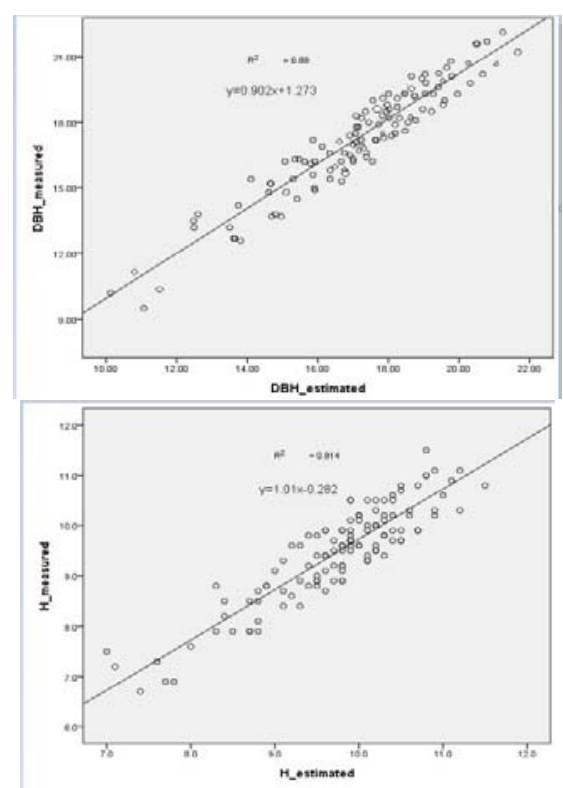

FIGURE III. REGRESSION ANALYSIS FOR DBH AND H

\section{CONCLUSION AND DISCUSSION}

In this study, through the application of the survey data as entry parameters for simulation system, computer technology to design and develop the visual simulation system for fir stand growth, the stand static and dynamic three-dimensional visual simulation is implemented. This study selects fir as the survey species, and applies 3PG model and the environmental factors to achieve its dynamic growth. As can be seen, 3PG model can be well to predict the growth of fir forest, and then get a more realistic expression tree growth, providing reliable support platform for forest resource managers. As for the model parameters are a lot, complex to get, and often obtained from other studies, so the 3PG model used in stand visualization system is limited to a certain extent. The relatively simple data requirements by reducing model parameters without the impact to physiological processes and growth forecast accuracy are crucial to the development of eco-physiological model and the efficiency of visualization System.

\section{ACKNOWLEDGMENT}

The paper was supported by National Natural Science Foundation of China (31170590) and National High Technology Research and Development Program 863 (2012AA102002). The paper's corresponding author is Huaiqing ZHANG.

\section{REFERENCES}

[1] B.Quan, L.Y.Tang, Visualization of Stand Growth in Virtual Geographic Environments.Journal of Fujian College of Forestry, 24(3), pp. 224-228, 2004

[2] B. Song,David J.M., Forest visualization at multiple scales for management and planning. Series E Technological Sciences, 49(1), pp. 27-34, 2006.

[3] Peter J D, Modeling growth and Water use in four Pinus Patulastands with the 3-PG model. Southern African Forestry Journal, 191, pp. 53-62,2001M.C. Guo, J.F. Liu, Modeling the stand growth of Chinese fir plantations. Journal of Fujian College of Forestry, 31(1), pp. 65-68, 2011.

[4] X.G. Chen,Accumulation and Growth Model of Cunninghamia lanceolata Plantation.Protection Forest Science and Technology,7(79), pp. 28-29, 2007.

[5] Almeida A C, Landsberg J J, Sands P J, Parameterisation of 3-PG for Fast Growing Eucalyptus Grandis Plantations. Forest Ecology and Management, 193(1-2), pp. 179-195, 2004.

[6] Coops N C, Waring R H, Landsberg J J, Estimation of potential forest productivity across the Oregon transect using satellite data and monthly weather records. International Journal of Remote Sensing, 22, pp. 3797-3812, 2001.

[7] K.N. Lu, H.Q. Zhang, Design and Implementation of Individual Tree Growth Visualization System of Cunninghamia lanceolata.Forest Research, 25(2), pp.207-211, 2012. 